\title{
factorplot: Improving Presentation of Simple Contrasts in Generalized Linear Models
}

by David A. Armstrong II

\begin{abstract}
Recent statistical literature has paid attention to the presentation of pairwise comparisons either from the point of view of the reference category problem in generalized linear models (GLMs) or in terms of multiple comparisons. Both schools of thought are interested in the parsimonious presentation of sufficient information to enable readers to evaluate the significance of contrasts resulting from the inclusion of qualitative variables in GLMs. These comparisons also arise when trying to interpret multinomial models where one category of the dependent variable is omitted as a reference. While considerable advances have been made, opportunities remain to improve the presentation of this information, especially in graphical form. The factorplot package provides new functions for graphically and numerically presenting results of hypothesis tests related to pairwise comparisons resulting from qualitative covariates in GLMs or coefficients in multinomial logistic regression models.
\end{abstract}

\section{Introduction}

The problem of presenting information about categorical covariates in generalized linear models is a relatively simple one. Nevertheless, it has received some attention in the recent literature. To be clear about the problem, consider the following linear model where $y$ is the dependent variable and $G=\{1,2, \ldots, m\}$ is a categorical independent variable that can be represented in the regression model by $m-1$ dummy regressors, each one representing a different category of $G$. The reference category, of course, is omitted. Thus, the model looks as follows:

$$
\begin{aligned}
& E\left(y_{i}\right)=\mu_{i} \\
& g\left(\mu_{i}\right)=\beta_{0}+\beta_{1} D_{i 1}+\beta_{2} D_{i 2}+\cdots+\beta_{m-1} D_{i m-1}+\beta_{m} X_{i 1}+\ldots+\beta_{m+k-1} X_{i k}+\varepsilon_{i},
\end{aligned}
$$

where $D_{i 1}=1$ if $G_{i}=1, D_{i 2}=1$ if $G_{i}=2$, etc. $X_{i k}$ represent an arbitrary set of additional variables of any type. Here, each of the coefficients on the dummy regressors for $G\left(\beta_{1}, \ldots, \beta_{m-1}\right)$ gives the difference in the conditional transformed mean of $y$ between the category represented by the dummy regressor and the reference category, controlling for all of the other $X_{i k}$. However, the $m-1$ coefficients for the categories of $G$ imply $\frac{m(m-1)}{2}$ simple contrasts representing every pairwise comparison between categories of $G$. Any single pairwise comparison of non-reference category coefficients can be conducted in a straightforward fashion. If the goal is to discern whether the conditional mean of $y$ given $G=1$ is different from the conditional mean of $y$ given $G=2$ holding all of the $X$ variables constant, the quantity of interest is:

$$
t=\frac{b_{1}-b_{2}}{\sqrt{V\left(b_{1}-b_{2}\right)}},
$$

where

$$
V\left(b_{1}-b_{2}\right)=V\left(b_{1}\right)+V\left(b_{2}\right)-2 V\left(b_{1}, b_{2}\right) .
$$

Thus, the calculation is not difficult, but calculating and presenting all of these differences can become cumbersome, especially as $m$ gets large. ${ }^{1}$ The problem comes not in the calculation of these quantities, but in the parsimonious presentation of this information that will allow users to evaluate any desired (simple) contrasts easily. Below, I discuss two extant methods used to present such information. Floating absolute risk (FAR) was first suggested by Easton et al. (1991) and was more rigorously justified, though with different estimation strategies, by Firth and De Menezes (2004); de Menezes (1999); Plummer (2004). FAR is a means of overcoming the reference category problem by calculating floating variances for all levels of a factor (including the reference category). These floating variances can be used to perform hypothesis tests or construct floating confidence intervals that facilitate the graphical comparison of different categories (i.e., [log-]relative risks). The multiple comparisons literature has traditionally been focused on finding the appropriate $\mathrm{p}$-values to control either the

\footnotetext{
${ }^{1}$ Tools to carry out these computations already exist in the multcomp package in R (Hothorn et al., 2008).
} 
family-wise error rate (e.g., Holm, 1979) or the false discovery rate (e.g., Benjamini and Hochberg, 1995) in a set of simultaneous hypothesis tests. Presentation of this information has either been in the form of line displays (e.g., Steel and Torrie, 1980) or compact letter displays (e.g., Gramm et al., 2006) with more recent innovation here by Graves et al. (2012).

However, when simple contrasts are the only quantities of interest, neither method above is perfect. When floating/quasi-variances are presented, the user still has to evaluate a potentially large number of hypothesis tests by either relying on the overlap in the floating confidence intervals or by calculating the floating t-statistic. Either solution requires a good deal of cognitive energy on the part of the analyst or reader. Compact letter displays do well at identifying patterns of statistical significance, but are perhaps cumbersome to investigate when patterns of (in)significance are complicated and, though mitigated to some degree, the problem still exists for the more recent multcompTs suggested by Graves et al. (2012). Below, I discuss a means for presenting this information in a manner that will permit the immediate evaluation of all the $m(m-1) / 2$ hypothesis tests associated with simple contrasts. The method I propose can also calculate analytical standard errors that are not prone to the same potential inferential errors produced by floating variances. I provide methods to summarize, print and plot the information in a way that is both visually appealing and straightforward to understand.

\section{Solutions to the reference category problem}

There are a number of reasonable solutions to the reference category problem. ${ }^{2}$ The first solution is to present all of the covariance information required to calculate $t$-statistics for contrasts of interest (i.e., the variance-covariance matrix of the estimators). This solution provides the reader with all necessary information to make inferences. However, it does not provide an easy way for all of these inferences to be presented. Another solution is to re-estimate the model with different reference categories in turn. ${ }^{3}$ This method produces the correct inferential information, but it is inelegant. The modal response to the reference category problem is a failure to do anything to discover (or allow readers to investigate) the implied pairwise differences not captured by the estimated coefficients.

Easton et al. (1991) proposed the idea of floating absolute risk as a means for evaluating multiple comparisons in matched case-control studies. The idea was to provide sufficient information such that readers could perform multiple comparisons with estimates of floating absolute risk at the expense of presenting a single extra number for each binary variable representing a level of a categorical covariate (i.e., risk factor). Although Greenland et al. (1999) disagreed on terminology and on the utility of Easton's idea of a floating scale, they agreed on the utility presenting information that would permit users to easily make the right inferences about relative risks among any levels of a categorical risk factor. Both Firth and De Menezes (2004) and Plummer (2004) provided a more rigorous statistical foundation on which to build estimates of floating absolute risk (or as Firth and De Menezes call them, quasi-variances). Firth and De Menezes' method has been operationalized in $\mathrm{R}$ in the qvcalc package (Firth, 2010) and both the methods of Plummer as well as Greenland et al. have been operationalized in the float () and ftrend() functions, respectively, in the Epi package (Carstensen et al., 2013). In general, these solutions allow sufficient (or nearly sufficient) information to be presented in a single column of a statistical table that makes valid, arbitrary multiple comparisons possible.

The measures of floating absolute risk are often used to create floating (or quasi-) confidence intervals. ${ }^{4}$ Presenting these intervals allows the user to approximately evaluate hypothesis tests about any simple contrast. While the exact nature of these confidence intervals is somewhat controversial (for a discussion, see Easton and Peto (2000); Greenland et al. $(1999,2000)$ ), all agree that confidence intervals can be profitably put around some quantity (either the log-relative risks versus the reference category or the floating trend) to display the uncertainty around these quantities and permit visual hypothesis tests.

The methods discussed above still require the analyst or reader to either evaluate the pairwise hypothesis tests based on the extent to which confidence intervals overlap or calculate the floating $\mathrm{t}$-statistic for each desired contrast. If the former, readers must still engage in a cognitive task of position detection (Cleveland, 1985) and then make an inference based on the extent to which intervals overlap. As the horizontal distance between vertically-oriented floating confidence intervals grows, this task becomes more difficult. Finally, as Easton et al. (1991) suggests, floating variances are a

\footnotetext{
${ }^{2}$ The problem here applies particularly to polytomous, unordered risk factors or covariates. The case of ordinal risk factors, where only the difference in adjacent categories is of interest, is a bit less troublesome and will not be dealt with separately here.

${ }^{3}$ In fact, this re-parameterization method could be used to deal with more complicated contrasts, too. For example, it could be used to deal with the problem proposed by Greenland et al. (1999) wherein they wanted to estimate the relative risk of being above a particular category on birthweight.

${ }^{4}$ Occasionally, quasi-variance estimates are negative, which provide the right inferences, but do not permit plotting of quasi-confidence intervals.
} 
"virtually sufficient" summary of the uncertainty relating to relative risks; however, they can produce erroneous inferences if the error rate is sufficiently high. Both Firth and De Menezes (2004) and Plummer (2004) provide methods for calculating this error rate, which is often small relative to other sources of error in the model.

To put a finer point on the problem, consider the example below using data from Ornstein (1976) from the car package (Fox and Weisberg, 2011). The model of interest is:

$$
\begin{aligned}
\text { Interlocks }_{i} & \sim \text { Poisson }\left(\mu_{i}\right) \\
\log \left(\mu_{i}\right) & =\beta_{0}+\beta_{1} \log _{2}\left(\text { Assets }_{i}\right)+\gamma \text { Sector }_{i j}+\text { 日Nation }_{i m}
\end{aligned}
$$

where $\gamma$ represents a set of coefficients on the $j=9$ non-reference category dummy variables for the 10 sectors represented in the data and $\boldsymbol{\theta}$ is the set of coefficients for the $m=3$ coefficients on the non-reference category dummy variables representing the four nations in the dataset. The goal is to determine which sectors (and/or nations) have significantly different transformed conditional means of Interlocks. The quasi-variances can be presented along with the coefficients permitting hypothesis testing at the reader's discretion. This approach is economical, but still requires the interested reader to make 27 pairwise hypothesis tests for sector and three pairwise hypothesis tests for nation, beyond those presented in the coefficient table.

The plot of the floating confidence intervals provides similar information, but readers are still required to make judgements about statistical significance with a visual method prone to occasional inferential errors. Consider Figure 1, which presents confidence intervals using the three different functions that produce floating variances $\mathrm{R}-\operatorname{qvcalc}()$, float () and ftrend(). ${ }^{5}$ In the figure, the floating confidence interval for the mining sector overlaps four other floating confidence intervals and does not overlap the remaining five intervals. ${ }^{6}$ Advice from Smith (1997) suggests that only confidence intervals not containing the point estimate against which the test is being done are significant. Here, all of the pairwise differences with the mining coefficient are significant because none of the point estimates are within the $95 \%$ confidence interval for mining. A more conservative strategy is to fail to reject null hypotheses where confidence intervals overlap and to reject otherwise. Using this criterion, the mining sector is different from five other coefficients - Agriculture, Banking, Construction, Finance and Wood. Browne (1979) shows that making inferences from confidence intervals requires a knowledge of the different sampling variances of the underlying random variables for which the confidence intervals have been constructed (i.e., the widths of the intervals matter); the decision does not rest solely on the extent to which the intervals overlap. While Browne's method may produce more appropriate inferences, it is hardly less work than producing the hypothesis tests directly. When the appropriate pairwise hypothesis tests are performed, without adjusting the $p$-values for multiple testing, it is clear that the mining coefficient is different from seven coefficients when using a two-sided test, as Table 1 shows.

Even if the evidence regarding the outcome of a hypothesis test from two confidence intervals is clear, there are other potential sources of error. Cleveland (1985) finds that detecting position along a common scale is one of the easiest tasks of graphical perception, but that discerning length is considerably more difficult. His experiments show that readers are prone to errors in even the easiest graphical perception tasks and the error rate is nearly twice as high when readers are asked to adjudicate the relative lengths of lines. Conducting hypothesis tests using confidence intervals is an endeavor rife with opportunities for inferential errors.

Means for calculating and presenting models with multiple simple contrasts have developed in the multiple testing literature as well. While the thrust of the literature mentioned above was dealing with the reference category problem directly, the multiple comparisons literature has placed greater focus on finding the appropriate $p$-values for a set of hypothesis tests rather than a single test. This can be accomplished through controlling the family-wise error rate (the probability of committing a Type I error on any of the tests in the set) or the false discovery rate (the proportion of falsely rejected hypotheses among those rejected). Chapter 2 of Bretz et al. (2011) provides a brief, but informative discussion of these general concepts. While these are useful concepts, and the package discussed below permits users to adjust $p$-values in a number of ways to address these issues, I am more interested in how the multiple testing literature has developed around the presentation of multiple pairwise comparisons.

\footnotetext{
${ }^{5}$ The figure below subtracts the arbitrary constant from the results of $f \operatorname{trend}()$ to put all of these estimates on the same scale. I recognize that this is not what the authors had intended, but this should not lead to erroneous inferences in any event (Easton and Peto, 2000).

${ }^{6}$ Horizontal gray lines have been drawn at the smallest lower- and largest upper-bounds of the mining sector floating confidence intervals to facilitate comparison. Note that differences across the three methods in the upper bounds and lower bounds were in the third decimal place.
} 
Table 1: Analytical Test of Differences between Mining (MIN) and Other Sectors

\begin{tabular}{lc} 
Contrast & Estimate/(SE) \\
\hline MIN - AGR & $0.250^{*}$ \\
& $(0.069)$ \\
MIN - BNK & $0.416^{*}$ \\
& $(0.084)$ \\
MIN - CON & $0.739^{*}$ \\
& $(0.210)$ \\
MIN - FIN & $0.361^{*}$ \\
& $(0.067)$ \\
MIN - HLD & $0.265^{*}$ \\
& $(0.118)$ \\
MIN - MAN & 0.128 \\
& $(0.071)$ \\
MIN - MER & $0.188^{*}$ \\
& $(0.085)$ \\
MIN - TRN & 0.098 \\
& $(0.071)$ \\
MIN - WOD & $-0.248^{*}$ \\
& $(0.072)$ \\
\hline
\end{tabular}

* $\mathrm{p}<0.05$, two-sided.

Estimates and standard errors produced by glht() from the multcomp package.

Gramm et al. (2006) discuss the two generally accepted methods for presenting multiple comparisons - the line display and the letter display. A line display (see for example, Steel and Torrie, 1980) prints a column where each row represents a single element in the multiple comparisons. In the example above, using the Ornstein data, these would be the names of the various sectors. Then, vertical lines are drawn connecting all values that are not significantly different from each other. This is a relatively simple display, but as shown generally by Piepho (2004) and in this particular case, it is not always possible to faithfully represent all of the pairwise comparisons with connecting line segments. Note that in the third line, a discontinuity is required to properly depict all of the pairwise relationships. Further, this method requires that the levels of factors (at least potentially) be reordered to identify insignificant differences. This reordering, while reasonable for unordered factors, is not at all reasonable if the factor is inherently ordered. Figure 2(a) shows the line display for the Ornstein model above. A compact letter display (Piepho, 2004) places a series of letters by each level of the categorical variable such that any two levels with the same letter are not significantly different from each other. ${ }^{7}$ Each letter essentially defines a set of factor levels that have insignificant differences in coefficients among them. For example, Banking, Construction and Finance all share the letter "a", which means their coefficients are statistically indistinguishable from each other. Note that Wood is the sole factor level with " $\mathrm{f}$ ", meaning that it has a statistically different coefficient than all of the other factor levels. These are more flexible than line displays, though they can still be improved upon. Even though these displays do identify all pairwise significant relationships, they do not immediately identify the sign and size of the differences and what appear to be complicated patterns of significance may appear more simple with a different mode of display.

Graves et al. (2012) discuss enhancements to the letter display that make it somewhat more visually appealing and make the cognitive tasks involved less cumbersome. This method is operationalized by the multcompTs function in the multcompView package. While these functions are potentially useful, they are A) still improved upon by the method discussed below and B) not intended for use directly with "glm" class objects or "glht" class objects. Despite the improvements over letter displays, complicated patterns of (in)significance still result in cluttered displays.

\footnotetext{
${ }^{7}$ The boxplot on the graph is a boxplot of the linear predictor from the statistical model. If there were no other covariates in the model, this would just be a boxplot of the response variable by the different factor levels. While this does provide some information, it does not indicate how the predicted response changes as a function of the factor holding other things constant, which would perhaps be more useful.
} 
Figure 1: Quasi-confidence Intervals for the Ornstein Model

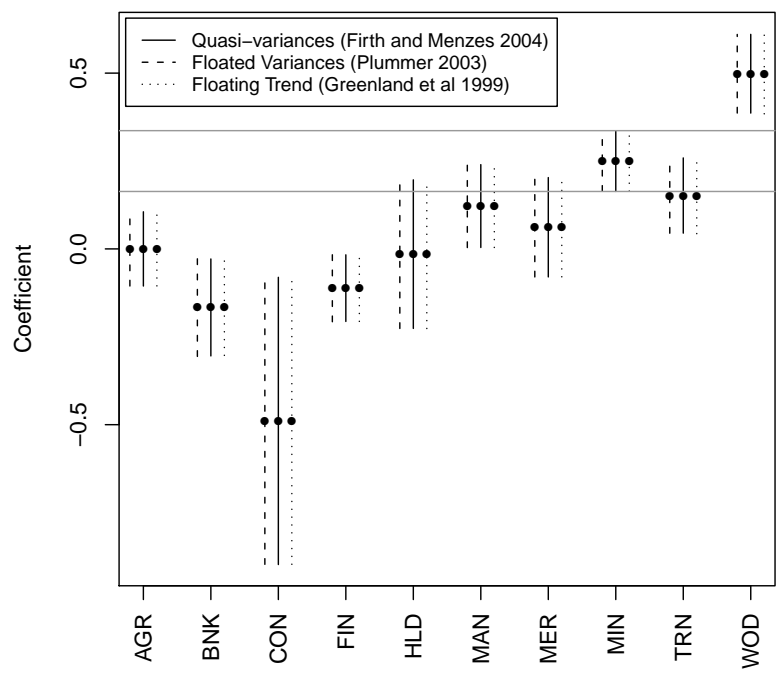

Figure 2: Line and Letter Displays for Ornstein Model

(a) Line Display

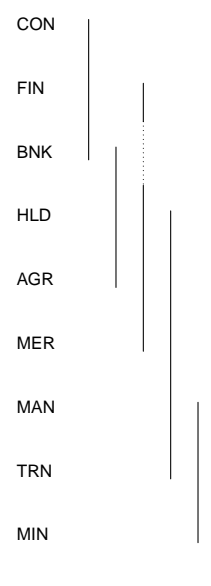

WOD (b) Letter Display

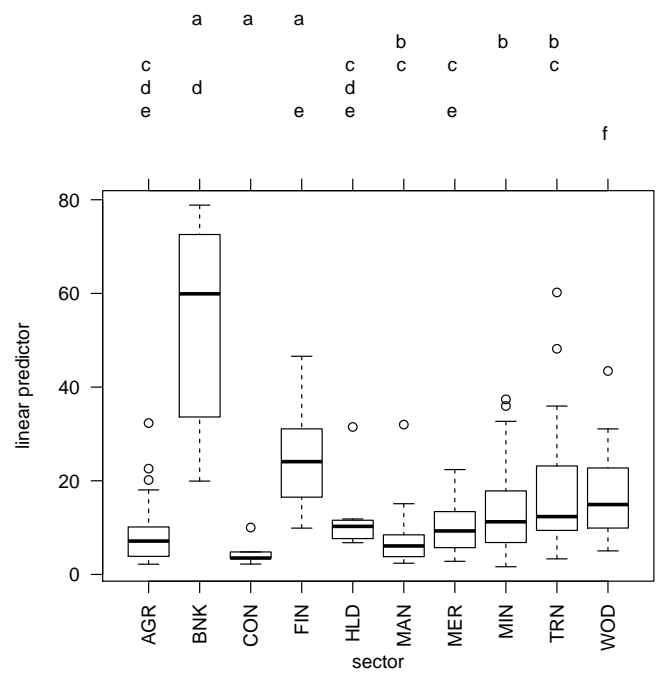




\section{An alternative method of presentation}

I argue that a good solution to the reference category problem is one that permits the most efficient presentation and evaluation of a series of hypothesis tests relating to various (simple) factor contrasts. As discussed above, both the numerical presentation of floating variances and the visual presentation of floating confidence intervals are not maximally efficient on either dimension (presentation or evaluation) when the analyst desires information about the simple pairwise difference between coefficients related to the levels of a factor (i.e., simple contrasts). Similarly, I suggested that compact letter displays (and to a lesser extent multcompTs), though they present all of the appropriate information, are not maximally efficient at presenting the desired information graphically. As Chambers et al. (1983) and Cleveland (1985) suggest, one efficient way of presenting many pairwise relationships is through a scatterplot matrix or a generalized draftsman's display (a lower- or upper-triangular scatterplot matrix). ${ }^{8}$ The important feature of a scatterplot matrix is the organization of pairwise displays in a common scale. Thus, a display that directly indicates the difference for the simple contrasts of interest would be superior to one that requires the user to make $(m(m-1)) / 2$ pairwise comparisons from $m$ floating variances or confidence intervals.

The factorplot function in the package of the same name (version 1.1) for R computes all pairwise comparisons of coefficients relating to a factor; its print, summary and plot methods provide the user with a wealth of information regarding the nature of the differences in these coefficients. ${ }^{9}$ These functions overcome the problems suffered by previous methods as they present the results of pairwise hypothesis tests directly in a visually appealing manner.

The function calculates equation (3) for each simple contrast directly through a set of elementary matrix operations. First, $\boldsymbol{d}$, a $m \times \frac{m(m-1)}{2}$ matrix in which each column has one entry equal to positive one, one entry equal to negative one and all the remaining entries equal to zero is created. The positive and negative ones indicate the comparison being calculated. Using the coefficients for the desired factor covariate (call them $g$, a row-vector of length $m$ ), I calculate $\Delta=g d$. Standard errors for contrasts are calculated using the $m$ rows and columns of the variance-covariance matrix of the estimators from the model (call this $V(g)$ ): $V(\Delta)=\boldsymbol{d}^{\prime} \boldsymbol{V}(g) \boldsymbol{d}$. The $\Delta$ vector and the square root of the diagonal of $V(\Delta)$ (both of length $\frac{m(m-1)}{2}$ ) are then organized into $(m-1) \times(m-1)$ upper-triangular matrices where the rows refer to the first $m-1$ elements of $g$ and the columns refer to the last $m-1$ elements of $g$. The entries indicate the difference between the coefficient represented by the row and the coefficient represented by the column and its standard error.

The function has methods for objects of class "lm", "glm", "glht" and "multinom" which do slightly different things depending on the input. The default method will accept a vector of estimates and either A) a full variance-covariance matrix or B) a vector of quasi or floated variances that will be turned into a diagonal variance-covariance matrix. The methods for "lm", "glm", "glht" and "summary.glht" objects calculate the pairwise differences in the linear predictor for the values of the specified factor variable. The method for "multinom" class objects calculates the pairwise differences in coefficients across the categories of the dependent variable for a single variable (i.e., column of the model matrix).

\section{Example 1: Ornstein data}

The factorplot method for "lm" class objects has six arguments. The first two arguments, obj and adjust.method, indicate the object and the method by which p-values are to be adjusted for multiple comparisons (possibilities include all of those to $\mathrm{p}$.adjust from the stats package). The factor. variable argument indicates the factor for which comparisons are desired. pval allows the user to set the desired Type I error rate and two. sided allows the user to specify whether the null hypothesis is tested against a one- or two-sided alternative with the latter as the default. The order argument sets the ordering of the coefficients, with three possibilities — 'natural', 'alph' and 'size'. The 'natural' option maintains the original ordering of the factor, the 'alph' option sorts them alphabetically and the 'size' option sorts in ascending order of the magnitude of the coefficient. The choices made here propagate through the plot, print and summary methods.

The plot method for "factorplot" class objects produces something akin to an upper-triangular scatterplot. The analogy is not perfect, but the idea is similar; each entry of the rows-by-columns display indicates the pairwise difference between coefficients. The statistical significance of these

\footnotetext{
${ }^{8}$ Cleveland (1985) makes the argument in favor of a full scatterplot matrix, but in this case, the information presented in the upper-triangle is sufficient as nothing new could be learned by examining the full square matrix.

${ }^{9}$ The methods for "lm", "glm", "multinom" and the default method use the calculations mentioned below. The method for "glht" and "summary.glht" objects uses the built-in functionality from the multcomp package to do these calculations. The benefit here is that if a small subset of comparisons is desired, this subset can be identified in the call to glht () and only those comparisons will be computed, thus increasing efficiency.
} 
differences is indicated by three colors (one for significant-positive, one for significant-negative and one for insignificant differences). The three colors can be controlled with the polycol argument and the text color within the polygons can be controlled with the textcol argument. ${ }^{10}$ The plot method also allows the user to specify the number of characters with which to abbreviate the factor levels through the abbrev.char argument. Setting this to an arbitrarily high value will result in no abbreviation. Finally, the trans argument allows the user to impose a post-hypothesis-test transformation to the coefficient estimates. For example, if the underlying model is a logistic regression, tests will be done on the log-relative risks, but the relative risks could be plotted with trans = "exp". ${ }^{11}$ By default, the function prints legends identifying the colors and numbers; these can be turned on or off with the logical arguments print.sig. leg and print. square.leg, respectively. Figure 3 shows the display for the Ornstein model. The following code produces the result in the figure.

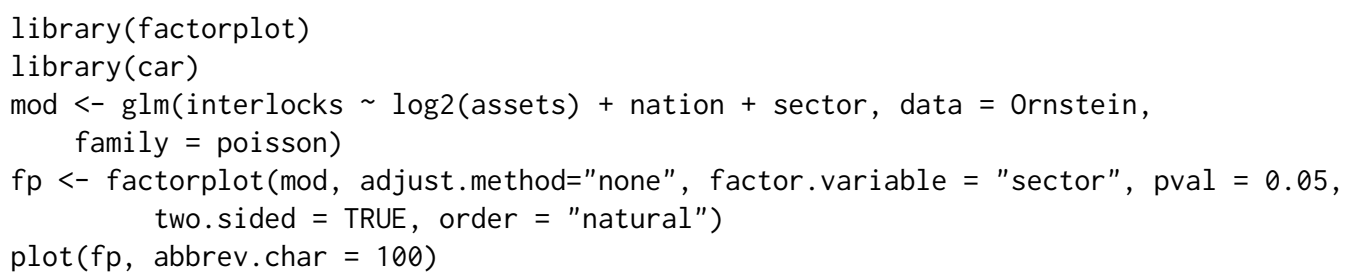

The print method for a "factorplot" object prints all of the pairwise differences, their accompanying analytical standard errors and (optionally adjusted) $p$-values. The user can specify the desired number of decimal places for rounding, with the digits argument. The sig argument is logical allowing the user to print all pairwise differences if FALSE and only significant differences when TRUE. The print method also permits the same trans argument as the plot method for objects of class "factorplot". An example of the output from the print method is below. Here, twenty-five of the forty-five pairwise differences are statistically different from zero when.

Figure 3: Plotted factorplot object for Ornstein model

\begin{tabular}{|c|c|c|c|c|c|c|c|c|c|}
\hline & $\sum_{m}^{y}$ & zo & $\underline{z}$ & 呈 & $\frac{z}{\Sigma}$ & $\stackrel{\frac{\pi}{山}}{\Sigma}$ & $\frac{z}{\Sigma}$ & 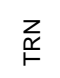 & ڤి \\
\hline AGR & $\begin{array}{r}-0.17 \\
0.10\end{array}$ & $\begin{array}{r}-0.49 \\
0.21\end{array}$ & $\begin{array}{r}-0.11 \\
0.08\end{array}$ & $\begin{array}{r}-0.01 \\
0.12\end{array}$ & $\begin{array}{l}0.12 \\
0.08\end{array}$ & $\begin{array}{l}0.06 \\
0.09\end{array}$ & $\begin{array}{l}0.25 \\
0.07\end{array}$ & $\begin{array}{l}0.15 \\
0.08\end{array}$ & $\begin{array}{l}0.50 \\
0.08\end{array}$ \\
\hline BNK & & $\begin{array}{c}-0.32 \\
0.22\end{array}$ & $\begin{array}{l}0.05 \\
0.07\end{array}$ & $\begin{array}{l}0.15 \\
0.13\end{array}$ & $\begin{array}{l}0.29 \\
0.10\end{array}$ & $\begin{array}{l}0.23 \\
0.11\end{array}$ & $\begin{array}{l}0.42 \\
0.08\end{array}$ & $\begin{array}{l}0.32 \\
0.08\end{array}$ & $\begin{array}{l}0.66 \\
0.09\end{array}$ \\
\hline CON & & & $\begin{array}{l}0.38 \\
0.22\end{array}$ & $\begin{array}{l}\mathbf{0 . 4 7} \\
0.23\end{array}$ & $\begin{array}{l}0.61 \\
0.21\end{array}$ & $\begin{array}{l}0.55 \\
0.22\end{array}$ & $\begin{array}{l}0.74 \\
0.21\end{array}$ & $\begin{array}{l}0.64 \\
0.22\end{array}$ & $\begin{array}{l}0.99 \\
0.22\end{array}$ \\
\hline FIN & & & & $\begin{array}{l}0.10 \\
0.12\end{array}$ & $\begin{array}{l}0.23 \\
0.08\end{array}$ & $\begin{array}{l}0.17 \\
0.09\end{array}$ & $\begin{array}{l}0.36 \\
0.07\end{array}$ & $\begin{array}{l}0.26 \\
0.07\end{array}$ & $\begin{array}{l}0.61 \\
0.08\end{array}$ \\
\hline HLD & & & & & $\begin{array}{l}0.14 \\
0.12\end{array}$ & $\begin{array}{l}0.08 \\
0.13\end{array}$ & $\begin{array}{l}0.26 \\
0.12\end{array}$ & $\begin{array}{l}0.17 \\
0.12\end{array}$ & $\begin{array}{l}0.51 \\
0.12\end{array}$ \\
\hline MAN & & & & & & $\begin{array}{l}-0.06 \\
0.09\end{array}$ & $\begin{array}{l}0.13 \\
0.07\end{array}$ & $\begin{array}{l}0.03 \\
0.08\end{array}$ & $\begin{array}{l}0.38 \\
0.08\end{array}$ \\
\hline MER & & & & & & & $\begin{array}{l}0.19 \\
0.09\end{array}$ & $\begin{array}{l}0.09 \\
0.09\end{array}$ & $\begin{array}{l}0.44 \\
0.09\end{array}$ \\
\hline MIN & & & & & & & & $\begin{array}{r}-0.10 \\
0.07\end{array}$ & $\begin{array}{l}0.25 \\
0.07\end{array}$ \\
\hline TRN & $\begin{array}{l}\square \mathrm{Sic} \\
\square \mathrm{No} \\
\square \mathrm{Sic}\end{array}$ & $\begin{array}{l}\text { ificantly } \\
\text { Signific } \\
\text { ificantly }\end{array}$ & & $\begin{array}{l}\text { bold }=b \\
\text { ital }=\mathrm{SE}\end{array}$ & $\begin{array}{l}b_{\text {row }} \\
b_{1}-b_{\text {row }}\end{array}$ & & & & $\begin{array}{l}0.35 \\
0.08\end{array}$ \\
\hline
\end{tabular}

$\operatorname{print}(f p, \operatorname{sig}=T)$

$$
\text { Difference SE p.val }
$$

\footnotetext{
${ }^{10}$ The printing of the estimates and standard errors can both be turned off with print. est $=$ FALSE and print. se = FALSE, respectively.

${ }^{11}$ After the hypothesis tests are done, a matrix named $r$. bdiff holds the coefficient differences. The transformation is done as follows: do.call (trans, list ( $r$.bdiff)), so only transformations amenable to this procedure will work.
} 


$\begin{array}{llll}\text { AGR - CON } & 0.489 & 0.213 & 0.023 \\ \text { CON - HLD } & -0.474 & 0.235 & 0.045 \\ \text { BNK - MAN } & -0.288 & 0.102 & 0.005 \\ \text { CON - MAN } & -0.611 & 0.215 & 0.005 \\ \text { FIN - MAN } & -0.233 & 0.082 & 0.005 \\ \text { BNK - MER } & -0.228 & 0.106 & 0.032 \\ \text { CON - MER } & -0.551 & 0.220 & 0.013 \\ \text { AGR - MIN } & -0.250 & 0.069 & 0.000 \\ \text { BNK - MIN } & -0.416 & 0.084 & 0.000 \\ \text { CON - MIN } & -0.739 & 0.210 & 0.001 \\ \text { FIN - MIN } & -0.361 & 0.067 & 0.000 \\ \text { HLD - MIN } & -0.265 & 0.118 & 0.026 \\ \text { MER - MIN } & -0.188 & 0.085 & 0.029 \\ \text { BNK - TRN } & -0.318 & 0.082 & 0.000 \\ \text { CON - TRN } & -0.641 & 0.217 & 0.004 \\ \text { FIN - TRN } & -0.263 & 0.070 & 0.000 \\ \text { AGR - WOD } & -0.498 & 0.076 & 0.000 \\ \text { BNK - WOD } & -0.665 & 0.095 & 0.000 \\ \text { CON - WOD } & -0.988 & 0.215 & 0.000 \\ \text { FIN - WOD } & -0.610 & 0.077 & 0.000 \\ \text { HLD - WOD } & -0.513 & 0.121 & 0.000 \\ \text { MAN - WOD } & -0.376 & 0.080 & 0.000 \\ \text { MER - WOD } & -0.437 & 0.090 & 0.000 \\ \text { MIN - WOD } & -0.248 & 0.072 & 0.001 \\ \text { TRN - WOD } & -0.346 & 0.081 & 0.000\end{array}$

The summary method for "factorplot" objects prints the number of coefficients that are significantly smaller than the one of interest and the number of coefficients larger than the one of interest for each level of the factor. While this is not a common means of presenting the results, this does nicely summarize the extent of significant differences among the coefficients. Below is an example of printout from the summary method. It is easy to see that the wood industry (WOD) has the highest conditional means as it is significantly bigger than all of other categories. It is also easy to see that the construction industry (CON) has one of the smallest conditional means as it is significantly smaller than seven of the other categories and not significantly bigger than any.

\begin{tabular}{lrrr}
\multicolumn{4}{c}{ summary (fp) } \\
sig+ & sig- & insig \\
AGR & 1 & 2 & 6 \\
BNK & 0 & 5 & 4 \\
CON & 0 & 7 & 2 \\
FIN & 0 & 4 & 5 \\
HLD & 1 & 2 & 6 \\
MAN & 3 & 1 & 5 \\
MER & 2 & 2 & 5 \\
MIN & 6 & 1 & 2 \\
TRN & 3 & 1 & 5 \\
WOD & 9 & 0 & 0
\end{tabular}

Together, the factorplot function and its associated print, plot and summary methods provide a wealth of information including direct hypothesis tests using analytical standard errors for the simple contrasts most commonly desired in (G)LMs.

\section{Example 2: H. pylori and gastric precancerous lesions}

Plummer et al. (2007) were interested in discerning the extent to which infection with H. pylori containing the cytotoxin-associated (cagA) gene increased the severity of gastric precancerous lesions. They found that cagA+ patients had increased risks of more severe lesions while cagA- patients were only at significantly higher risk (than their uninfected counterparts) of chronic gastritis. Table 2 summarizes the results of the relative risk of the various types of gastric lesions versus the baseline of normal or superficial gastritis.

The default method for the factorplot function allows the user to supply a vector of point estimates and (floating) variances rather than an estimated model object. This function will be particularly useful for those scholars in epidemiology, where floating standard errors are more routinely presented. With 7 levels of the factor in Table 2, there are 21 pairwise comparisons implied, which would require 
Table 2: Results from Plummer et al. (2007)

\begin{tabular}{lcc|cc} 
& \multicolumn{2}{c}{ cagA- } & \multicolumn{2}{c}{ cagA+ } \\
& OR & FSE & OR & FSE \\
\hline Normal and superficial gastritis & 1.00 & 0.242 & 1.00 & 0.320 \\
Chronic gastritis & 2.12 & 0.096 & 4.33 & 0.101 \\
Chronic atrophic gastritis & 1.44 & 0.156 & 3.89 & 0.160 \\
Intestinal metaplasia I & 1.31 & 0.140 & 4.14 & 0.141 \\
Intestinal metaplasia II & 1.44 & 0.380 & 10.8 & 0.349 \\
Intestinal metaplasia III & 1.46 & 0.484 & 21.9 & 0.431 \\
Dysplasia & 0.90 & 0.375 & 15.5 & 0.311 \\
\hline
\end{tabular}

$\mathrm{OR}=$ odds ratio

FSE = floating standard error

Adapted from Figure 1 in Plummer et al. (2007, p1331).

users to do a lot of calculations. However, inputting the estimates and floated variances into $\mathrm{R}$ and subjecting them to the factorplot function can do all of the calculations automatically. Below is an example of how the results could be used in conjunction with the factorplot suite of functions.

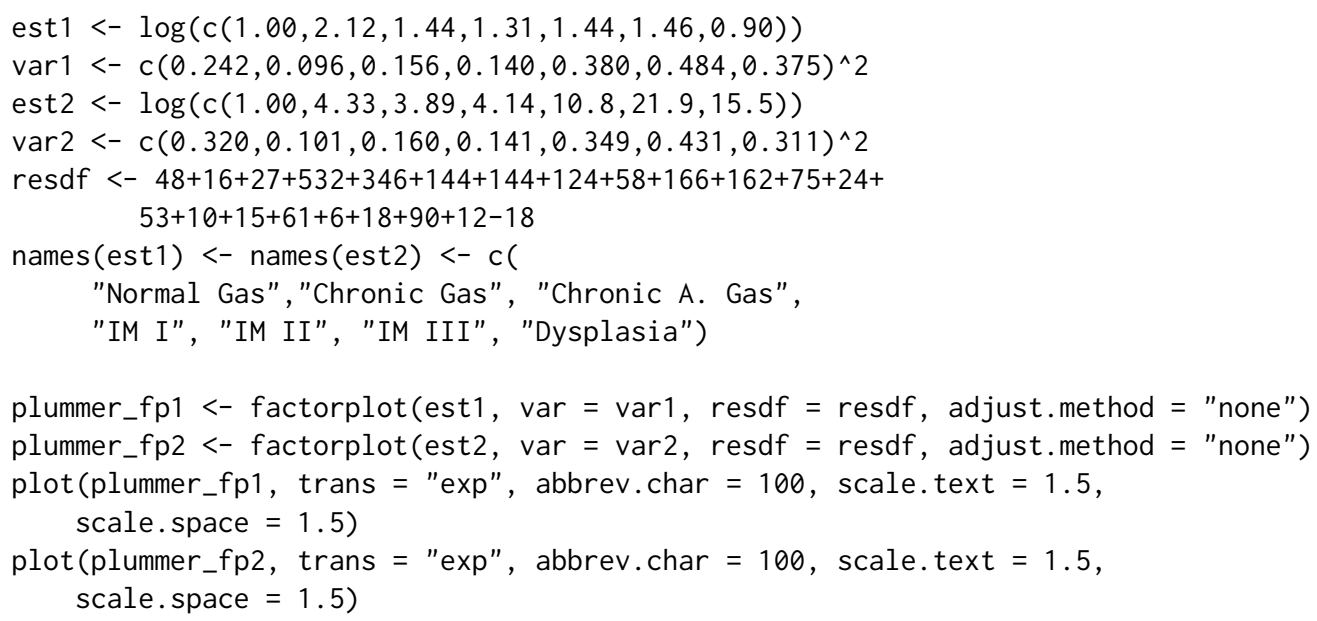

The plots are displayed in Figure 4. The left-hand plot suggests that H. pylori cagA- seems to raise the risk of chronic gastritis relative to Intestinal metaplasia I and the reference group of normal and superficial gastritis. The differences in the risk of chronic gastritis and chronic atrophic gastritis or dysplasia are also significant. The right-hand plot indicates that there are no significant differences among the second through fourth diagnoses and the fifth through seventh diagnoses. The difference between the risk of intestinal metaplasia I and II (for cagA+) is also significant.

\section{Example 3: vote choice in France}

When factorplot () encounters an object of class multinom, it will make comparisons within the same variable across all levels of the dependent variable. The coefficient table presents a specific set of pairwise comparisons - namely those indicating the relationship of each variable to the binary choice of each non-reference category versus the reference category. However, other comparisons implied by that coefficient table may be interesting or useful and should be investigated.

In the example below, I estimate a multinomial logistic regression model of vote choice (vote) on a number of standard controls: retrospective national economic evaluations (retnat), self-placement on the left-right ideological continuum (lrself), gender (male) and age (age). ${ }^{12}$

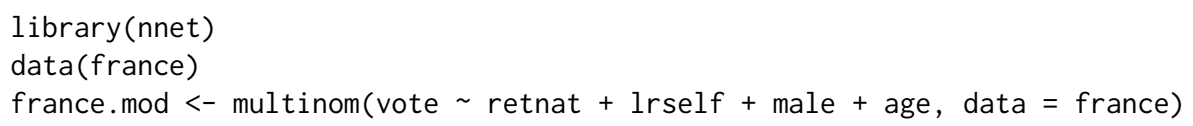

${ }^{12}$ See help for france in the package factorplot for more details about the origin and coding of the data. 
Figure 4: Results from Plummer et al. (2007) Presented as factorplots

(a) cagA-

\begin{tabular}{|c|c|c|c|c|c|c|}
\hline & 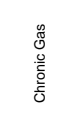 & 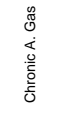 & $\bar{\Sigma}$ & $\underline{\underline{\Xi}}$ & 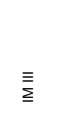 & $\begin{array}{l}\frac{\mathrm{w}}{\mathrm{m}} \\
\frac{\mathrm{m}}{\mathrm{m}} \\
\frac{1}{2}\end{array}$ \\
\hline Normal Gas & $\begin{array}{l}2.12 \\
0.26\end{array}$ & $\begin{array}{l}1.44 \\
0.29\end{array}$ & $\begin{array}{l}1.31 \\
0.28\end{array}$ & $\begin{array}{l}1.44 \\
0.45\end{array}$ & $\begin{array}{l}1.46 \\
0.54\end{array}$ & $\begin{array}{l}0.90 \\
0.45\end{array}$ \\
\hline Chronic Gas & & $\begin{array}{l}0.68 \\
0.18\end{array}$ & $\begin{array}{l}0.62 \\
0.17\end{array}$ & $\begin{array}{l}0.68 \\
0.39\end{array}$ & $\begin{array}{l}0.69 \\
0.49\end{array}$ & $\begin{array}{l}0.42 \\
0.39\end{array}$ \\
\hline Chronic A. Gas & & & $\begin{array}{l}0.91 \\
0.21\end{array}$ & $\begin{array}{l}1.00 \\
0.41\end{array}$ & $\begin{array}{l}1.01 \\
0.51\end{array}$ & $\begin{array}{l}0.62 \\
0.41\end{array}$ \\
\hline$|\mathrm{M}|$ & & & & $\begin{array}{l}1.10 \\
0.40\end{array}$ & $\begin{array}{l}1.11 \\
0.50\end{array}$ & $\begin{array}{l}0.69 \\
0.40\end{array}$ \\
\hline IM II & & & & & $\begin{array}{l}1.01 \\
0.62\end{array}$ & $\begin{array}{l}0.62 \\
0.53\end{array}$ \\
\hline IM III & $\begin{array}{l}\text { S Sigit } \\
\text { 品 } \\
\text { Nig }\end{array}$ & & $\begin{array}{l}\text { lld }=\mathrm{b}_{\text {oll }}- \\
=\mathrm{S}\left(\mathrm{b}_{\mathrm{col}}\right.\end{array}$ & & & $\begin{array}{l}0.62 \\
0.61\end{array}$ \\
\hline
\end{tabular}

(b) $\operatorname{cag} \mathrm{A}+$

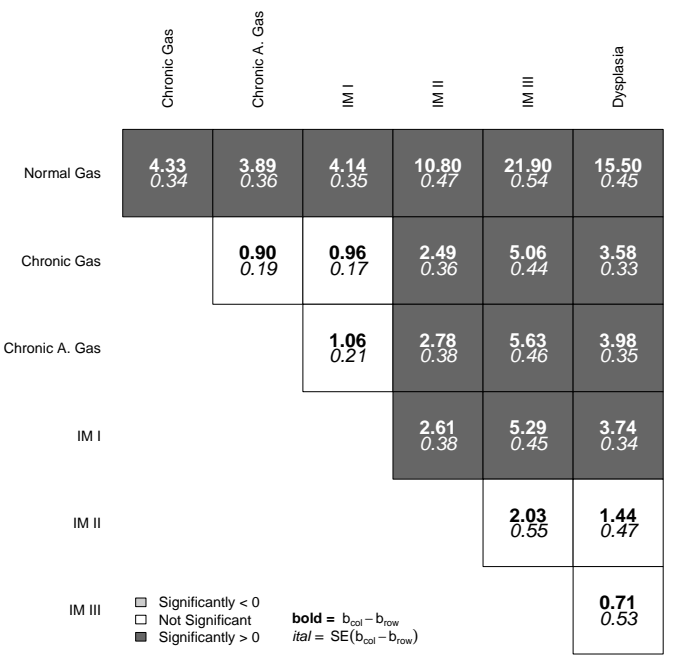

fp3 <- factorplot (france.mod, variable = "age")

$\operatorname{plot}(\mathrm{fp} 3)$

Figure 5 shows that as people get older, they are more likely to vote for RPR or UDF than the Greens or Communists (PCF) and more likely to vote for the Socialists (PS) than the Greens. If one is interested in whether variables have significant effects on vote choice, all pairwise comparisons should be considered. factorplot makes it easy for users to appropriately evaluate all relevant pairwise comparisons.

\section{Conclusion}

Easton's (1991) contribution of floating absolute risk has been influential, especially in epidemiology and medicine, allowing researchers to present easily information that permits the reader to make any pairwise comparison among the different levels of a risk factor. Firth and De Menezes (2004); de Menezes (1999) and Plummer (2004) have provided not only a rigorous, model-based foundation for this idea, but have also provided software that easily produces these quantities for a wide array of statistical models. I argue that while these quantities are interesting and useful, floating confidence intervals, which are often provided ostensibly to permit hypothesis testing can be imprecise and potentially misleading, as regards hypothesis testing. Compact letter displays (Piepho, 2004) are a step in the right direction, but I argue that they can still be improved upon in terms of graphically presenting information of interest to many researchers. In the common situation wherein one is interested in simple contrasts, the factorplot () functions and their associated print, plot and summary methods discussed above provide much greater transparency with respect to the presentation and evaluation of hypothesis tests than floating absolute risk or quasi-variance estimates. The visual presentation of direct hypothesis tests requires much less effort to adjudicate significance and uncover patterns in the results than other methods, including compact letter displays. While the calculation of these hypothesis tests is not novel, the methods of presenting and summarizing the information represent a significant advance over the previously available general solutions available in R.

\section{Acknowledgements}

I would like to thank Bob Andersen, Ryan Bakker, John Fox, Bill Jacoby, Martyn Plummer and an anonymous reviewer as well as participants in the Regression III course at the ICPSR Summer Program for helpful comments and suggestions. 
Figure 5: Plotted factorplot object for Age from Multinomial Logit model

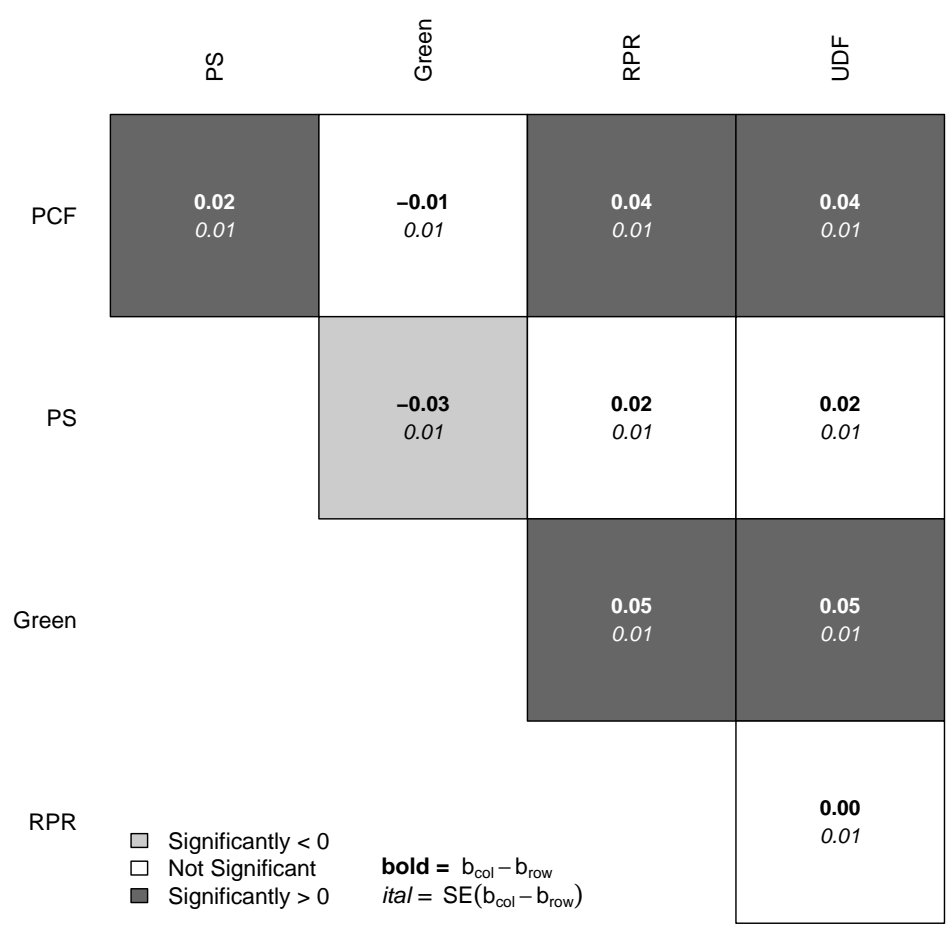

\section{Bibliography}

Y. Benjamini and Y. Hochberg. Controlling the false discovery rate: A practical and powerful approach to multiple testing. Journal of the Royal Statistical Society Series B, 57(1):289-300, 1995. [p5]

F. Bretz, T. Hothorn, and P. Westfall. Multiple Comparisons Using R. Taylor and Francis Group, LLC, Boca Raton, FL, 2011. [p6]

R. H. Browne. Visual assessment of the significance of a mean difference. Biometrics, 35(3):657-665, 1979. [p6]

B. Carstensen, M. Plummer, E. Laara, and M. Hills. Epi: A Package for Statistical Analysis in Epidemiology, 2013. URL http://CRAN. R-project.org/package=Epi. R package version 1.1.44. [p5]

J. M. Chambers, W. S. Cleveland, B. Kleiner, and P. A. Tukey. Graphical Methods for Data Analysis. Wadsworth \& Brooks/Cole Publishing Company, Pacific Grove, CA, 1983. [p9]

W. Cleveland. Elements of Graphing Data. Wadsworth, Inc., Monterey, CA, 1985. [p5, 6, 9]

R. X. de Menezes. More Useful Standard Errors for Group and Factor Effects in Generalized Linear Models. $\mathrm{PhD}$ thesis, Department of Statistics, University of Oxford, 1999. [p4, 13]

D. F. Easton and J. Peto. Presenting statistical uncertainty in trends and dose-response relationships (letter). American Journal of Epidemiology, 152(393), 2000. [p5, 6]

D. F. Easton, J. Peto, and A. Babiker. Floating absolute risk: An alternative to relative risk in survival and case-control analysis avoiding an arbitrary reference group. Statistics in Medicine, 10:1025-1035, 1991. [p4, 5]

D. Firth. qucalc: Quasi variances for factor effects in statistical models, 2010. URL http: //CRAN. R-project. org / package=qvalc. [p5]

D. Firth and R. X. De Menezes. Quasi-variances. Biometrika, 91(1):65-80, 2004. [p4, 5, 6, 13]

J. Fox and S. Weisberg. An R Companion to Applied Regression. Sage, Thousand Oaks CA, second edition, 2011. [p6] 
J. Gramm, J. Guo, F. Hüffner, R. N. B, H. peter Piepho, and R. Schmid. Algorithms for compact letter displays: Comparison and evaluation. Computational Statistics $\mathcal{E}$ Data Analysis, 52(12):725-736, 2006. $[\mathrm{p} 5,6]$

S. Graves, H.-P. Piepho, L. Selzer, and S. Dorai-Raj. multcompView: Visualizations of Paired Comparisons, 2012. URL http://CRAN. R-project.org/package=multcompView. R package version 0.1-5. [p5, 7]

S. Greenland, K. B. Michels, and J. M. Robins. Presenting statistical uncertainty in trends and doseresponse relations. American journal of ..., 149(12):1077-1086, 1999. [p5]

S. Greenland, K. Michels, C. Poole, and W. Willett. Four of the authors reply [re: "presenting statistical uncertainty in trends and dose-response relationships"] (letter). American Journal of Epidemiology, 152:394, 2000. [p5]

S. Holm. A simple sequentially rejective multiple test procedure. Scandinavian Journal of Statistics, 6 : 65-70, 1979. [p5]

T. Hothorn, F. Bretz, and P. Westfall. Simultaneous inference in general parametric models. Biometrical Journal, 50:346-363, 2008. [p4]

M. Ornstein. The boards and executives of the largest canadian corporations. Canadian Journal of Sociology, 1:411-437, 1976. [p6]

H. Piepho. An algorithm for a letter-based representation of all pairwise comparisons. Journal of Computational and Graphical Statistics, 13:456-466, 2004. [p7,13]

M. Plummer. Improved estimates of floating absolute risk. Statistics in Medicine, 23:93-104, 2004. [p4, $5,6,13]$

M. Plummer, L. van Doorn, S. Franceschi, B. Kleter, F. Canzian, J. Vivas, G. Lopez, D. Colin, N. Muñoz, and I. Kato. Helicobacter pylori cytotoxin-associated genotype and gastric precancerous lesions. Journal of the National Cancer Institute, 99:1328-1334, 2007. [p11, 12]

R. W. Smith. Visual hypothesis testing with confidence intervals. Proceedings of the $22^{n} d$ SAS User's Group, pages 1252-1257, 1997. [p6]

R. Steel and J. Torrie. Principles and Procedures of Statistics. McGraw-Hill, New York, 1980. [p5, 7]

David A. Armstrong II

University of Wisconsin - Milwaukee

Department of Political Science

P.O. Box 413

Milwaukee, WI 53201

United States of America

armstrod@uwm. edu 